\title{
Sistemas urbanos y la competencia por el conocimiento. \\ Publicaciones y patentamientos científicos en Chile, 2002-2015
}

Aarón Napadensky Pastene

Doctor en Arquitectura y Estudios Urbanos por la Pontificia Universidad Católica de Chile. Director del Laboratorio de Estudios Urbanos, académico e investigador del Doctorado en Arquitectura y Urbanismo y el Departamento de Planificación y Diseño Urbano. Facultad de Arquitectura, Construcción y Diseño, Universidad del Bío Bío, Chile.

\section{Daniela Villouta}

Estudiante magíster en Urbanismo, Universidad de Chile, arquitecto por la Universidad del Bío Bío, Chile, y ayudante de investigación del Doctorado en Arquitectura y Urbanismo de la misma universidad. villoutadaniela@gmail.com.

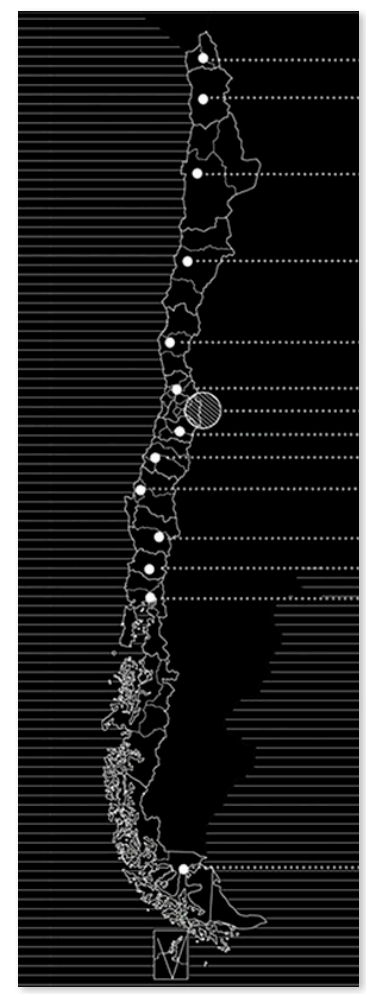

http://dx.doi.org/10.30972/crn.24242926 ISNN 1666-6186. Volumen 24 - N. ${ }^{\circ} 24$ (Junio de 2018)- Pp. 173-196 - Recibido: 09-06-17. Aprobado: 19-03-18. 


\title{
Resumen
}

El presente trabajo se aproxima a la relación entre sistemas urbanos (FUA) y producción/ aplicación de conocimiento, definidos por publicaciones y patentamientos científicos. Para ello se realiza un ranking que establece seis categorías de sistemas urbanos, desde súper cumbres a valles, a partir de cuatro indicadores: (i) población; (ii) producto interno bruto; (iii) artículos científicos publicados y (iv) solicitudes de patentamientos. Los datos levantados se evaluaron a partir de estadística descriptiva básica, calculando rangos en tres niveles mediante desviación estándar y cuadros de control - esto para todos los datos en columnas-, que posteriormente se cruzaron. El resultado fue una cronología del movimiento de los principales FUA nacionales en relación con los indicadores planteados, que identifica tendencias hacia su especialización, tanto de la producción de conocimiento como de su aplicación. Así también se identificaron cambios en la primacía de Santiago, capital del país, en relación con los FUA intermedios. Finalmente se debaten y abren nuevas interrogantes sobre la relación entre sistemas urbanos, competitividad y conocimiento.

\section{Palabras clave}

Economía del conocimiento; sistemas urbanos; publicaciones y patentamientos científicos.

\begin{abstract}
Urban systems and the competition to control knowledge. Publications and scientific patents in Chile, 2002-2015.

The present paper approaches the relationship between Functional Urban Areas (FUAs), and production/application of knowledge, as defined by scientific publications and patenting trends. To do this, a ranking of six categories of FUA was established, from super summits to valleys, based on four indicators: (i) population; (ii) gross domestic product; (iii) published scientific articles; and (iv) requests for patent applications. The data collected were evaluated from basic descriptive statistics, calculating ranges in three levels by standard deviation and control tables. All the data was presented in columns which were subsequently comparatively analyzed. The result was a chronology of the dynamics of the main national FUAs in relation to the selected indicators, identifying trends toward specialization both in the production of knowledge and its use, as well as changes in the primacy of Santiago, the capital of the country, in relation to intermediate FUAs. Finally, new questions about the relationship between the FUAs' urban qualities are raised and debated in terms of urban systems, competitiveness and knowledge.
\end{abstract}

\section{Keywords}

Knowledge economy; urban systems; scientific publications and scientific patents. 


\section{Introducción*}

En las últimas décadas del siglo XX, la economía norteamericana atravesó por una de sus más profundas recesiones, contexto en el cual la derecha radical de aquel momento sentenció a la planificación urbana como la culpable, al inhibir el libre funcionamiento del mercado (Hall, 1996; Pérez, 2004; Harvey, 2007, 2008). Sin embargo, ya pasado tiempo, es posible decir que esta recesión evidenció las inequívocas turbulencias y transformaciones propias de una transición desde una economía industrial a una de servicios, cuestión que al amparo de la expansión neoliberal fue reajustando mercados laborales y jerarquías urbanas.

La visión neoliberal y el consiguiente cambio productivo no tardaron en llegar a Latinoamérica y promover desregulación, privatización y retirada del Estado (MEYER \& BäHR, 2001), permitir en una primera instancia que se aumentaran flujos de capital internacional y la participación de actores privados en la economías locales, replicar en las grandes ciudades del continente las tendencias de países industrializados; desplomar industrias manufactureras, intensificar nuevas industrializaciones primarias, al tiempo que las economías urbanas se tercerizaban (AguILAR, 2002), creando emergentes distritos de servicios avanzados o re-cualificando los cascos fundacionales (Chion, 2002; PARnReiter, 2002, 2005, 2011; López Levi, 1997; LudeÑa, 2002; JanoschKa, 2002; De Mattos, 2010; Ciccolella, 1999; Ducci, 2002; NAPADENSKY, 2014, 2016), dando cuenta de una nueva relación entre urbes, economía y conocimiento. Así, reestructuración productiva y tercerización económica, iniciada en un contexto de desregulación y apertura, fueron el telón de fondo sobre el cual se ha discutido la idea de competitividad urbana, concepto que levanta escepticismo y adherencia (Fuentes, 2012). Dentro de estas últimas, destacan, por su vínculo entre ciudad y competitividad, las ideas de crear ambientes atractivos para conquistar talento calificado, capaz

\author{
* Este artículo es \\ resultado de un seminario \\ de investigación de la \\ Universidad del Bío Bío, \\ financiado con Fondos \\ Concursables de Innovación \\ Tecnológica de la Región \\ del Bío Bío. Corporación de \\ Fomento, CORFO e INNOVA \\ Bío Bío, más el apoyo de la \\ Dirección de Investigación \\ y Escuela de Graduados de \\ la Universidad del Bío Bío, \\ Chile.
}


de generar y aplicar conocimiento, cuestión fundamental para competir en un ambiente de reestructuración productiva, aumentando las posibilidades de generar innovación y promover mayor crecimiento económico local (Moliní y EstradA, 2015; Rodríguez-Pose y Comptour, 2012). Sin embargo, a nivel continental, la competitividad y sus estrategias siguen ancladas a la atracción de inversión, incentivos tributarios y subvenciones. En su gran mayoría carecen de una dimensión urbana y metropolitana (GonzÁLEz, 2012; MÉNDEZ ET ÁL., 2006; 2012).

Así, la discusión se centra en el caso de Chile, y cómo se han tendido —o no—a especializar y mover los sistemas urbanos nacionales intermedios en relación con Santiago, capital nacional, y primado centro de servicios terciarios de alto nivel (Escolano ET ÁL., 2007; Escolano \& OrTiz, 2010), esto en términos de publicaciones científicas como indicador parcial de producción de conocimiento, y solicitudes de patentamiento como indicador parcial de aplicación de conocimiento. La hipótesis de trabajo plantea que con la lógica de una emergente economía del conocimiento, las brechas entre la capital y los sistemas urbanos intermedios se ampliaron y profundizaron.

\section{Objetivo}

El objetivo central del presente trabajo es revisar y discutir en una primera y gruesa aproximación, en el marco del cambio que están experimentando las matrices productivas y bases laborales de los principales sistemas urbanos nacionales, de industrial a servicios intensivos en conocimiento, si la generación de artículos científicos, como un indicador parcial de producción de conocimiento, y las solicitudes de patentamientos científicos, como un indicador parcial de aplicación de conocimiento, evidencian una profundización en la primacía de Santiago sobre los sistemas urbanos intermedios, o si, por el contrario, es posible apreciar un estrechamiento en dicha distancia, ya sea por un mejor desempeño y especialización de estos últimos o por una pérdida de competitividad de la capital nacional. Para abordar lo planteado, se caracterizan los principales sistemas urbanos nacionales, tanto en población como Producto Interno Bruto (PIB), y se elabora un ranking a partir de estos indicadores y de su producción en publicaciones y solicitudes de patentamiento científico, además de presentar una breve panorámica de cómo se han movido en los últimos años, localizando y definiendo posiciones, identificando tendencias y anomalías. 


\section{Propuesta metodológica}

El método de caracterización y evaluación de los sistemas urbanos implicó su delimitación y homologación con las Functional Urban Areas, FUA ${ }^{1}$ (OCDE, 2013), la selección de aquellas que coincidían, en parte o totalmente, con capitales regionales, y el relevo de datos en cuatro cortes temporales: 2002, 2007, 2012 y 2015. La recolección, integración, análisis, interpretación y discusión de la información levantada se ordenó en tres etapas: $i$ ) recopilación bases de datos, solicitudes de patentamientos, publicaciones científicas, Producto Interno Bruto (PIB) y población; ii) generación y aplicación de una clasificación a escala país que identifica, en los términos propuestos, la tendencia a producir o aplicar conocimiento por parte de los FUA y iii) interpretación de resultados sobre la base de datos críticos, como inversión pública en investigación, población, tendencia a la especialización y distanciamiento de Santiago.

Las bases de datos consultadas fueron: (i) Instituto Nacional de Propiedad Industrial (INAPI), del Ministerio de Economía, Fomento y Turismo de Chile; (ii) revistas científicas, WOS, SCIELO y noSCIELO, en la Comisión Nacional de Ciencia y Tecnología (CONICYT), del Ministerio de Economía de Chile, y (iii) el Instituto Nacional de Estadísticas de Chile (INE). En la interpretación de resultados, también se puso sobre la mesa la capacidad de los FUA para capturar inversión pública en investigación; se utilizaron datos de CONICYT, y sus programas (i) Fondo nacional de Desarrollo Científico y Tecnológico (FONDECYT), (ii) Fondo de Equipamiento Científico y Tecnológico (FONDEQUIP); (iii) Programa de Atracción e Inserción de Capital Humano Avanzado (PAI); Fondo Nacional de Investigación y Desarrollo en Salud (FONIS); Programa de Investigación Asociativa (PIA); Fondo de Fomento al Desarrollo Científico y Tecnológico (FONDEF); Fondo de Financiamiento de Centros de Investigación en Áreas Prioritarias (FONDAP).

Para el cálculo del PIB de cada FUA, se tomó el PIB regional, se obtuvo el per cápita y se lo multiplicó por los habitantes de la FUA, tomando la población como dato constante, dado que su aumento se consideró marginal para el arco de tiempo en revisión. Para el cálculo PIB se usaron bases de datos del Banco Central de Chile (BCC). Tanto para parangonar los datos económicos del PIB como los de inversión pública en investigación, se tomaron las circulares presupuestarias de cada año de la Dirección de Presupuesto (DIPRES), del Ministerio de Hacienda de Chile.
1. FUA, acrónimo de Functional Urban Areas, categorización de la Organización para la Cooperación y el Desarrollo Económicos (OCDE, 2013) para definir áreas urbanas compuestas por más de una comuna, pero funcionalmente relacionadas. 


\section{Cuadro 1}

\section{Síntesis indicadores}

\begin{tabular}{ll}
\hline $\begin{array}{l}\text { Variable [desagregado por FUA } \\
\text { y años; 2002, 2007, 2012, 2015] }\end{array}$ & Fuente base de datos \\
\hline $\begin{array}{l}\text { Solicitudes de patentamientos en Chile } \\
\text { Artículos científicos publicados }\end{array}$ & $\begin{array}{l}\text { INAPI. Ministerio de Economía, } \\
\text { Fomento y Turismo de Chile }\end{array}$ \\
\hline Población & CONICYT. Ministerio de Educación de Chile \\
\hline PIB & CENSO 2002, INE, OCDE 2008 \\
\hline Inversión pública en investigación & INE; BCC \\
\hline
\end{tabular}

Fuente: elaboración propia

2. La información resultante se reagrupó y representó de manera secuencial, lo que permitió establecer con rapidez y claridad el movimiento de las FUA en las categorías y períodos $\bar{X}=\frac{\sum x}{n}$ establecidos, es decir, cómo fue su desempeño en términos de generación de publicaciones y solicitudes de patentamientos científicos, y su coincidencia con la concentración de inversión pública en investigación (cuadro 1).

3. Para el procesamiento de datos se utilizó un análisis de datos univariados, registrando las $S_{X}=\sqrt{\frac{1}{n-1} \sum_{i=1}^{n}\left(x_{i}-\bar{x}\right)^{2}}$ características, una a una, mediante medidas de tendencia central o media ${ }^{2}$, y de dispersión de datos o desviación estándar muestral típica ${ }^{3}$, registrando el promedio aritmético de fluctuación de estos respecto de su punto central. Sobre la base de la desviación estándar muestral de los datos analizados, se compararon los valores obtenidos y se los agrupó en tres rangos, para lo cual se utilizó análisis de control estadístico como método gráfico para evaluar la evolución de las FUA en cada variable, estableciendo límites de control, identificando con claridad aquellas FUA que están en la media, media superior o inferior y las que escapan a los límites de control, con lo cual se obtuvo una rápida y gráfica comparación cronológica (2002-2007-2012-2015). Este método constituye una aplicación adaptada de los 
sistemas de control de procesos, que representa sintéticamente las variaciones de un determinado proceso, identifica posibles coyunturas que escapan al orden más estructural de los fenómenos, y actúa gráficamente con rangos que determinan los datos que responden a la estructura del fenómeno, esto sobre la base de la variación aleatoria esperada de este (JuRAN, Godfrey, Hoogstoel, \& Schilling, 1999).

En última instancia, se elabora una categorización de FUA sobre la base de la clasificación de Florida (2009). La categorización corresponde a la siguiente: (i) Súper Cumbres, sobre la media, es decir, por encima del límite superior del rasgo estructural, en solicitudes de patente y publicaciones; (ii) Cumbres, sobre o igual a la media en solicitudes de patentes y publicaciones; (iii) Emergentes productoras de conocimiento, menor a la media en solicitudes de patentes y mayor igual a la media en publicaciones; (iv) Emergentes aplicadoras de conocimiento, mayor o igual a la media en solicitudes de patentes y menor a la media en publicaciones; $(v)$ Cumbres en desarrollo, menor a la media en patentes y solicitudes de publicaciones, pero mayor o igual a la media en población y mayor en PIB, y (vi) Valle, menor a la media en solicitudes de patentes, publicaciones y población e igual o menor en PIB (cuadro N. ${ }^{\circ}$ 2).

\section{Cuadro 2}

\section{Categorización sistemas urbanos}

\begin{tabular}{|c|c|c|c|c|c|c|}
\hline \multirow[t]{2}{*}{ N. ${ }^{\circ}$} & \multirow[t]{2}{*}{ Sigla } & \multirow[t]{2}{*}{ Categorías FUA } & \multicolumn{4}{|c|}{ Característica (según media del indicador) } \\
\hline & & & Sol. Patentes & Publicaciones & POB & PIB \\
\hline 1 & $\mathrm{SC}$ & Súper cumbre & $>*$ & $>*$ & - & - \\
\hline 2 & $\mathrm{CU}$ & Cumbres & $\geq$ & $\geq$ & - & - \\
\hline 3 & $\mathrm{EP}$ & Emergentes productoras de conocimiento & $<$ & $\geq$ & - & - \\
\hline 4 & EA & Emergentes aplicadoras de conocimiento & $\geq$ & $<$ & - & - \\
\hline 5 & $\mathrm{CD}$ & Cumbres en desarrollo & $<$ & $<$ & $\geq$ & $>$ \\
\hline 6 & VA & Valle & $<$ & $<$ & $\leq$ & $\leq$ \\
\hline
\end{tabular}

Fuente: elaboración propia (sobre la base de clasificación de ciudades de Florida, 2009) 
No obstante lo idónea, replicable y simple que se considera la metodología, esta tiene limitantes insoslayables. La primera es que la información que se utiliza proviene exclusivamente de bases de datos oficiales, asumiendo eventuales sesgos y parcialidades. En cuanto a la completitud de lo que se aborda aquí, es necesario consignar que los datos son parciales y unidimensionales, y no dan cuenta de la complejidad, naturaleza, magnitud o alcance geográfico de las dinámicas de producción y aplicación de conocimiento, menos de sus tendencias locacionales intraurbanas o de la relación con el desarrollo productivo territorial y el acervo local. Por otro lado, es una metodología simplificada y evidentemente cuantitativa y exploratoria, que excluye toda aproximación o explicación cualitativa, histórica, cultural, política o social que pueda comparecer en la comprensión de los resultados que arroja, pero abre camino para discutir sobre ello, especialmente cuando se identifican FUA que estando en condiciones de estar ahí, en la media, se encuentran fuera del rasgo estructural.

\section{Sistemas urbanos, creatividad y conocimiento}

La ciudad —o como se ha dado en llamar aquí, sistema urbano o FUA, reconociendo con ello la complejidad alcanzada por las urbes contemporáneas- ha sido desde siempre reducto de creatividad, innovación y conocimiento, lo que se rubrica en el auge que ha tenido la investigación y profundización de esta relación, cuya más reciente data se inicia con el fin occidental de la Revolución Industrial y posterior surgimiento del concepto de economía creativa, legitimada en la política pública cultural de fines del siglo XX en Australia y Gran Bretaña (Australia Creative Nation, 1994; Industries Mapping Document, Department of Culture, Media and Sports. DCMS, 1998), que enfatiza el papel del capital humano, las redes sociales y la creatividad de profesiones específicas en el desarrollo y competencias de mercado (Throsby, 2004, 2008).

En respuesta a la obsolescencia industrial urbana y la necesidad de restructuración de las grandes capitales industriales, surge el concepto de ciudad creativa, combinación de economía cultural e industria creativa (JURATE, 2011), que en tanto definición tiene tres componentes básicos, los cuales según su énfasis constituyen enfoque. El primero es la ciudad en tanto espacio de aplicación de herramientas creativas para el desarrollo urbano, idea que defiende la necesidad de una cultura de la creatividad, embebida dentro de actores y operadores urbanos, consolidando burocracias creativas que encaucen y legitimen el uso de la imaginación dentro de la esfera pública, privada y comunitaria (Landry, 2008; Darchen \& Temblay, 
2014). Un segundo énfasis es la ciudad como lugar de aglomeración y concentración de industrias creativas ${ }^{4}$ y servicios intensivos en conocimiento, o su acrónimo inglés KIS, Knowledge Intensive Services ${ }^{5}$ (García, 2008; Vence, 2007; Vence \& Rodil, 2003; Martinelli \& Cavola, 2002; Muller \& Zenker, 2001; Costas, 2008; Pratt, 2008), y el tercer enfoque es la urbe y su capacidad de atraer recursos humanos con competencias creativas o también llamados profesionales creativos o clase súper creativa ${ }^{6}$ (FLoRIDA, 2009, 2010, 2011).

Si bien, y en términos generales, la literatura suele coincidir en lo beneficioso de la concentración espacial de talento y creatividad para el surgimiento de actividades y servicios innovadores (Florida, 2010, 2011; Pérez y Marmolejo, 2008; SÁnchez, 2012), también se plantea que la relación entre crecimiento económico y concentración de personas creativas es poco clara (Hoyman \& Faricy, 2009; JuRATE, 2011), y que la adopción del paradigma de ciudad creativa tendería a favorecer funciones de alto nivel, todo dentro de un orden social neoliberal (BRENNER \& THEODORE, 2002; HARveY, 2005) y a consagrar ciertos estilos de vida de determinadas elites urbanas por sobre otros (РесK, 2005; KRäTKE, 2011), y que en último caso, esta discusión entre creativos y no creativos es una simplificación reduccionista y taxonómica (Howkins, 2001; Sсотт, 2006; РRATт, 2008).

\section{Casos de estudio: Chile}

Chile, en el concierto sudamericano, se caracteriza por una economía abierta y de corte neoliberal, de estructura política centralizada y presidencial, con un sistema de ciudades (Fig. 1) altamente primado, donde Santiago, capital del país, concentra casi la mitad de la población nacional y la mayor parte de los servicios avanzados a la producción (Escolano ET ÁL., 2007). La primacía de la FUA capital no solo es poblacional, sino también funcional, esto pese a que algunas regiones profundizaron su producción-especialización e incrementaron sus servicios comerciales de apoyo (Escolano \& OrTiz, 2010).

Transitando el primer cuarto del siglo XXI, es posible apreciar - no sin contradicciones e inestabilidades - una consolidación de las FUA intermedias, que, incididas tanto por su origen exportador o industrial como por la liberalización económica iniciada con el régimen militar (1973), evidencian —en consonancia con las narrativas académicas imperantes- marcadas tendencias a la desindustrialización y expansión de sus economías de servicios, fuertemente vinculadas con los nuevos procesos territoriales de industrialización neo-extractivista. No obstante lo anterior, no está claro si el reordena-
4. En una traducción, más literal que de fondo, las industrias creativas fueron clasificadas por HowKINS (2007) de la siguiente manera: i) Innovación más desarrollo, I+D; ii) Sector editorial; iii) Programas informáticos; iv) TV y radio; v) Diseño; vi) Música; vii) Cine; viii) Juegos y juguetes; ix) Publicidad; x) Arquitectura; xi) Artes escénicas; xii) Video juego; xiii) Moda y xiv) Arte.

5. El sistema estadístico europeo (EUROSTAT, 2014) clasifica los KIS en servicios intensivos en conocimiento de alta tecnología; servicios de mercado intensivo en conocimiento; servicios financieros intensivos en conocimiento y otros servicios intensivos en conocimiento.

6. Los profesionales creativos estarían compuestos por puestos de alta dirección, empresa y finanzas, sector jurídico, profesiones sanitarias y técnicas, ventas alto nivel y gestión ventas. La clase súper-creativa engloba a (i) informática y matemáticas; (ii) arquitectura y distintas ingenierías; (iii) ciencias sociales, físicas y de la vida; (iv) educación, enseñanza y lectura; (v) arte, diseño, entretenimiento deporte $y$ medios de comunicación. El mismo autor define una categoría (FLORIDA, 2009, 2010). 


\begin{tabular}{|lr|}
\hline Capitales & \multicolumn{1}{|c|}{ Total } \\
Nacionales & \multicolumn{1}{c|}{ Población } \\
\hline [A] Santiago & 5.428 .590 \\
[B] Buenos Aires & 15.625 .084 \\
[C] Monte Video & 3.286 .314 \\
[D] Asunción & 540.000 \\
[E] La Paz & 789.585 \\
[F] Brasilia & 2.562 .963 \\
[G] Lima & 9.605 .042 \\
[H] Quito & 2.100 .000 \\
[I] Bogotá & 6.763 .000 \\
[J] Caracas & 5.905 .463 \\
[K] Georgetown & 354.964 \\
[L] Paramaribo & 244.946 \\
[M] Cayena & 102.089 \\
& \\
\hline & \\
\hline Functional & Total \\
Urban Areas. & Población \\
\hline [1] Arica & 185.268 \\
[2]Iquique & 166.204 \\
[3]Antofagasta & 296.905 \\
[4]Copiapó & 129.901 \\
[5]La Serena & 333.472 \\
[6]Valparaiso & 880.063 \\
[7]Santiago & 6.566 .184 \\
[8]Rancagua & 298.184 \\
[9]Talca & 218.634 \\
[10]Concepción & 938.239 \\
[11]Temuco & 358.851 \\
[12]Valdivia & 140.559 \\
[13]Puerto Montt & 175.938 \\
[14]Punta Arenas & 119.496 \\
& \\
\hline
\end{tabular}

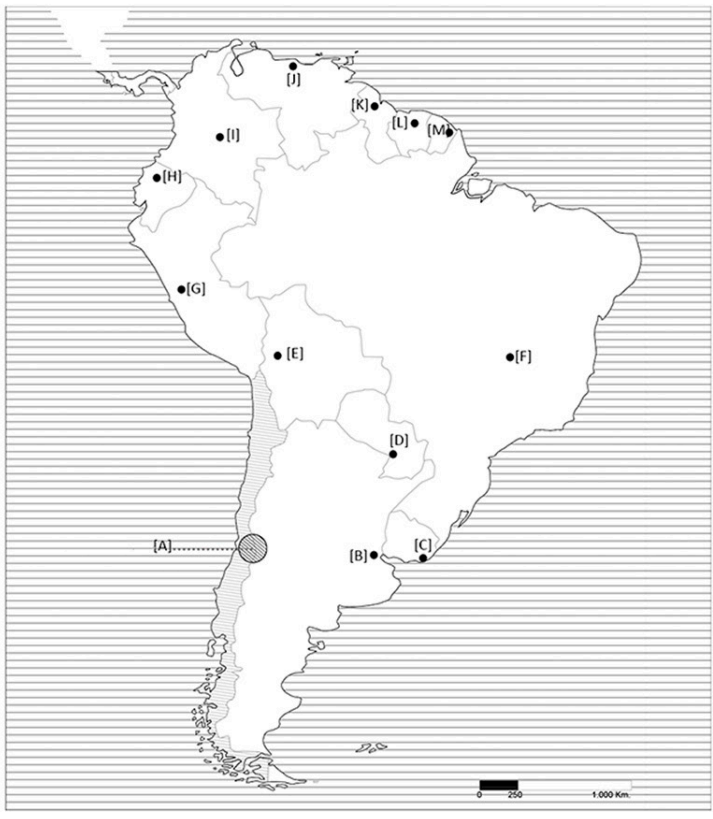

Mapa América del Sur, caso de estudio, Chile.



Mapa ubicación Functional Urban Areas en Chile. Capital Nacional: Santiago

\section{SIMBOLOGÍA}

Mapa América del Sur [A] Santiago; Capital nacional / Población: 17,72 Millones/hab.

Mapa ubicación Capitales Regionales - [1] Arica [2] Iquique [3] Antofagasta [4] Copiapó [5] La Serena [6] Valparaiso [7] Santiago [8] Rancagua[9]

Talca, [10] Concepción[11] C. Regional: Temuco [12] Valdivia [13] Puerto Montt [14] Punta Arenas

Figura 1.

Functional Urban Areas; FUA, en Chile, 2013.

Fuente: elaboración propia sobre la base de clasificación de Functional Urban en Chile por la OCDE, 2013 miento funcional que ha soportado, interactuado y coproducido la consolidación de las FUA intermedias - y también por cierto de la capital nacional- estaría generando, en términos de producción y búsqueda de nuevas aplicaciones al conocimiento, una concentración, aumentando la distancia entre estos y la capital nacional, o desconcentrando, acortando la brecha.

\section{PIB v/s atracción de recursos públicos para investigación}

En cuanto al PIB de las FUA, se establece como una medida aproximada de su dinamismo económico y poblacional. Así se identificaron cuatro puntas significativas, que pese 
a algunas variaciones de año en año, se han mantenido, y que en orden de importancia y jerarquía son Santiago, Antofagasta, Valparaíso y Concepción. Es significativo el caso de Antofagasta, que en el período de análisis alcanza el segundo lugar, aunque en población está por debajo de Valparaíso, Concepción, e incluso de La Serena o Temuco, cuestión explicada en gran medida por el auge minero local. También se aprecia cómo la distancia entre Santiago y las otras FUA nunca ha dejado de crecer (Fig. 2).

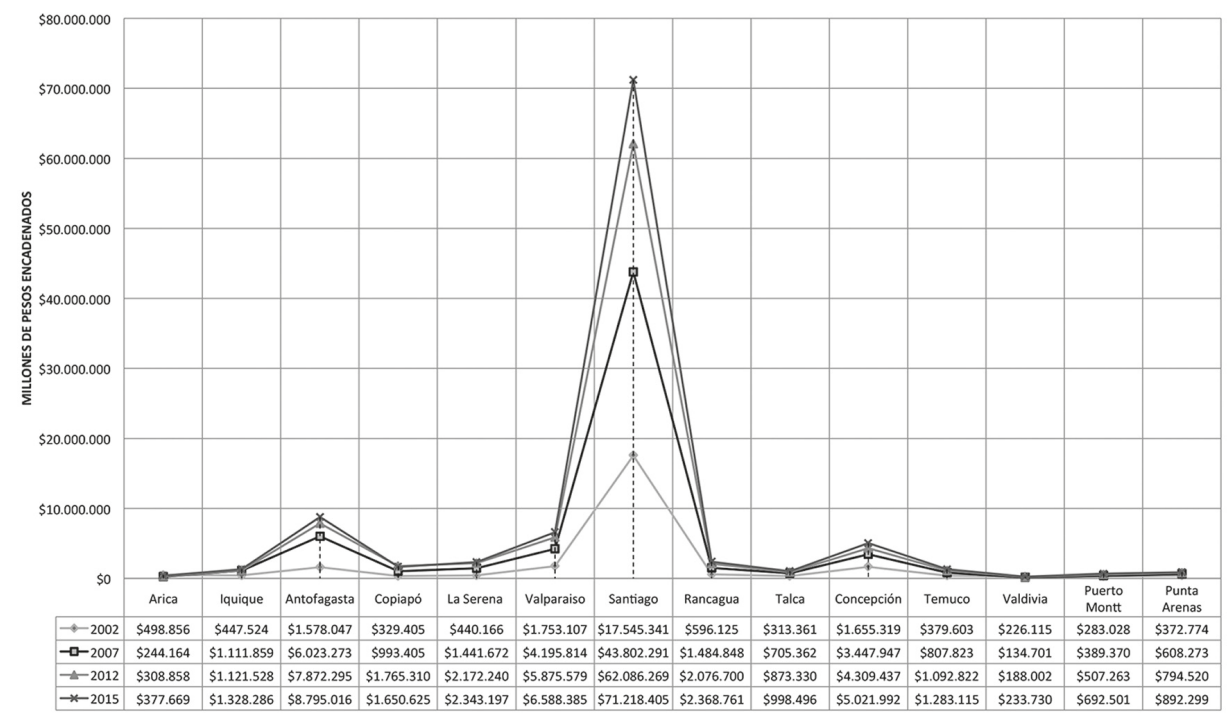

Figura 2.

Gráfico Producto Interno Bruto.

Fuente: elaboración propia sobre la base de datos estadísticos 2002-2015.

Banco Central de Chile, Cuentas Nacionales

Para el caso de la atracción de recursos públicos para investigación, no existe un correlato directo con la evolución del PIB, pero reitera la tendencia de distanciamiento entre la capital nacional y las FUA intermedias. Aunque estas últimas dan cuenta de un mejor desempeño en atraer recursos para investigación, palidecen frente a Santiago, especialmente en los últimos años, cuando esta inversión pública crece porcentualmente mucho más que el PIB (Fig. 3). También se pueden apreciar importantes fluctuaciones en cada período; es así que, por ejemplo, entre 2007 y 2012, si bien se produce un aumento en casi todas las FUA, Santiago y Concepción registran los mayores aumentos, lo que no 
vuelve a registrarse entre 2012 y 2015, cuando Santiago se escapa de forma importante. También se registran aumentos significativos, tanto en términos absolutos como porcentuales, en Temuco y Talca, pero especialmente en Valparaíso y Valdivia. La excepción es Concepción, que decrece en la atracción de fondos.

Figura 3.

Gráfico inversión pública en investigación. Fuente: elaboración propia sobre la base de fondos adjudicados por proyecto, Comisión Nacional de Investigación Científica Tecnológica, CONICYT, programas FONDECYT, FONDEF, FONIS, PIA, PAI, FONDEQUIP, FONDAP años 2002-2015

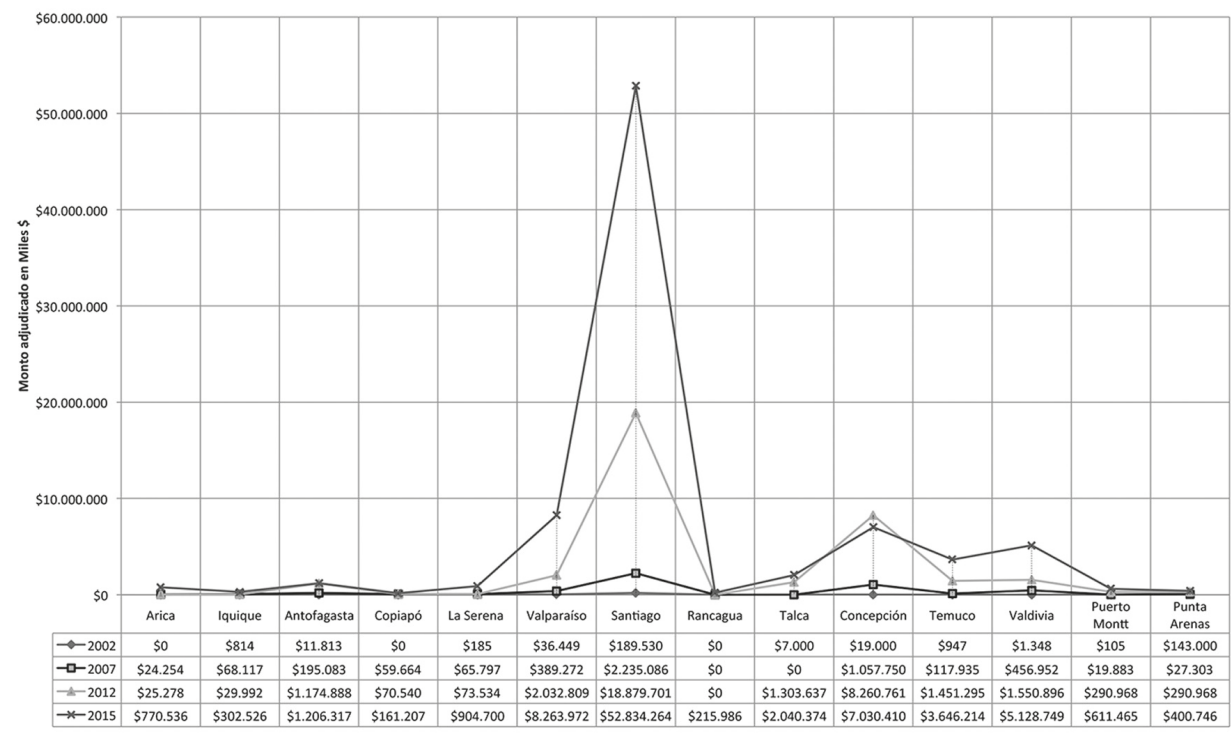

En 2012, Concepción estaba holgadamente sobre Valparaíso y no muy distante de Santiago, que aun así duplicaba. Finalmente, en 2015 Santiago consolida su distanciamiento y queda el orden en jerarquía de mayor a menor, con la FUA de Santiago en primer lugar, al cual le siguen muy por debajo Valparaíso, Concepción, Valdivia, Temuco, Talca, Antofagasta y La Serena.

\section{FUA, producción y aplicación de conocimiento}

En cuanto a la producción de conocimiento, medida parcialmente en publicaciones científicas, la tendencia da cuenta de una primacía de Santiago por sobre las FUA intermedias, cuestión que se intensifica con el tiempo; sin embargo, aparece Antofagasta como 
una de las puntas significativas y de mayor aumento en términos porcentuales, siempre en relación con su propia evolución. Así, en orden jerárquico quedan Santiago, Valparaíso, Concepción, Temuco, Valdivia, Antofagasta y Arica (Fig. 4).

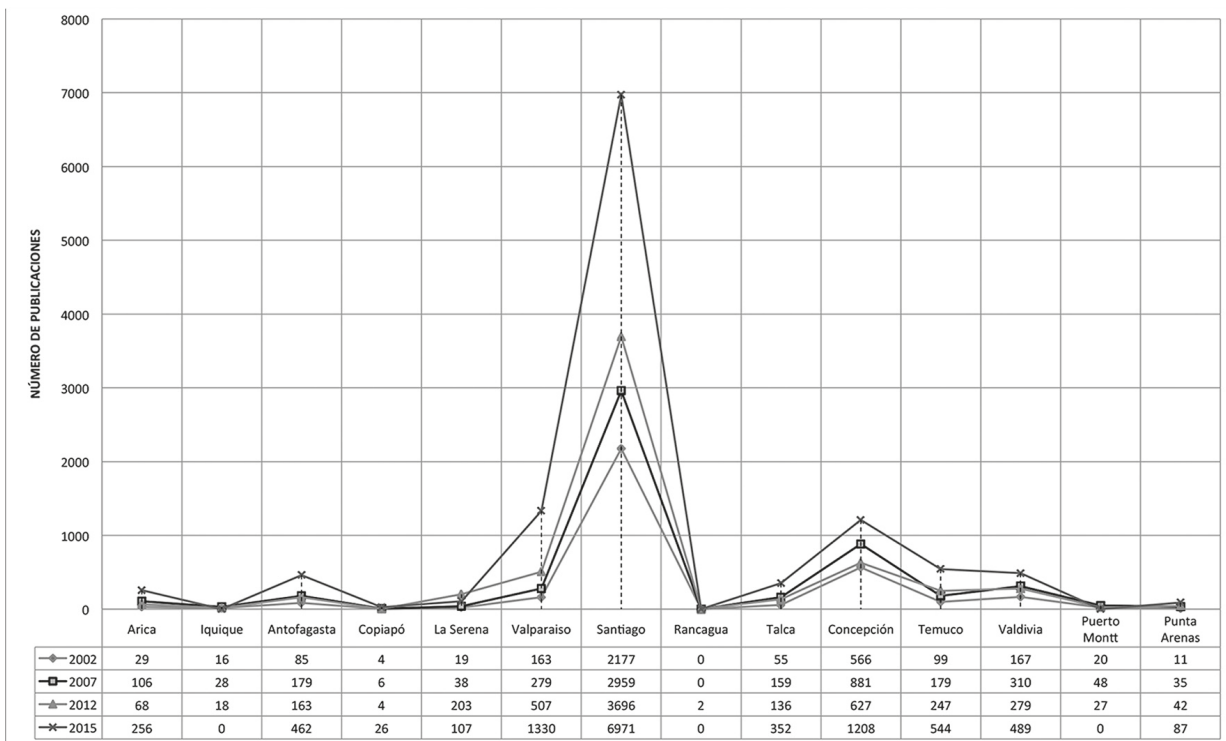

Si bien es esperable la correlación entre inversión pública en investigación y producción de publicaciones científicas, cuando se comparan ambos factores y se toma en cuenta el tamaño poblacional, el desempeño de Santiago tiende a relativizarse e incluso a disminuir, esto en relación con las FUA intermedias, donde sucede lo contrario. Por ejemplo, caucionando que los fondos públicos para investigación suelen ejecutarse en los años posteriores, a partir de una operación matemática simple se puede establecer que el promedio de publicaciones científicas por habitante para el año 2015 es el siguiente: Santiago y Concepción 0.001; Antofagasta y Temuco, 0.002 y Valdivia, 0.003. El costo de cada publicación, sobre la base del dato de inversión pública en investigación aquí pre-
Figura 4.

Gráfico publicaciones científicas. Fuente: elaboración propia sobre la base de Indicadores de Investigación por Clasificación, IES 20022015. Consejo Nacional de la Educación, CNDE 
sentado es el siguiente: Valdivia, \$10.488.239; Santiago, \$7.579.151; Temuco, \$6.702.599; Concepción \$5.819.875 y Antofagasta, \$2.611.075. Tres FUA en el año 2015 arrojan 0 para este indicador: Iquique, Rancagua y Puerto Montt, lo que más allá de la exactitud del dato marca una tendencia preocupante en el desempeño de producción de conocimiento.

En términos de la búsqueda de nuevas aplicaciones de conocimiento, medida parcialmente en solicitudes de patentes, el territorio nacional es más puntiagudo, siempre liderado por Santiago, seguido muy por debajo por Concepción y Valparaíso (Fig. 5). Lo relevante es el ascenso de una punta nueva, Puerto Montt, que en términos de buscar nuevas aplicaciones de conocimiento aparece empatando el cuarto lugar con Antofagasta. Si bien ambas tienen una débil atracción de recursos públicos para investigación, están en activos territorios de economías primarias extractivas, acuícola y minera, respectivamente, por lo que puede desprenderse, respecto de los datos analizados, que la industria local empieza a generar procesos spin-off utilizando conocimiento práctico local y posiblemente externo. Esta hipótesis se debe pesquisar con profundidad.

\section{Cruces y resultados}

Una vez caracterizadas las FUA, se procesaron los datos y se calculó la desviación estándar para cada ítem y año, en mérito de la variable y corte de análisis, se identificó en cada caso el rasgo estructural o área media y se completó con dichos resultados el cuadro 2. Pese a que la tabla-ranking oculta detalles, es lo suficientemente sincrética como para identificar cambios. Es posible decir que en el tramo analizado, las FUA de la zona central presentan mayor estabilidad, coincidiendo con el territorio desarrollado tempranamente por el primer auge exportador del siglo XIX; Santiago no varía como Súper cumbre, lo mismo para Valparaíso y Concepción, como Cumbres. Por su parte, Talca y Temuco se sostienen como Emergente productora de conocimiento y Rancagua como Cumbre en desarrollo (Fig. 6). Donde hay mayor inestabilidad en el período de análisis es en las zonas norte y sur. En el extremo norte, Arica e Iquique subieron de Cumbres en desarrollo a Emergente productora de conocimiento; Antofagasta pasó de ser Emergente productora de conocimiento a Cumbre; Copiapó, de Valle a Cumbre en desarrollo, para luego volver a Valle; La Serena, de Valle a Emergente productora de conocimiento; Valdivia se mantuvo en esa categoría, no así Puerto Montt, que parte de Valle, luego asciende a Emergente productora de conocimiento, vuelve a descender a Valle, para finalmente ascender a Emergente aplicadora de conocimiento, en sintonía con su condición de polo productivo industrial. 


\section{Sistemas urbanos y la competencia por el conocimiento.}

Publicaciones y patentamientos científicos en Chile, 2002-२०15



Figura 5 .

Gráfico solicitudes de patentes de invención.

Fuente: elaboración propia sobre la base de Expediente de Solicitudes de Patentes de invención del Instituto Nacional de Propiedad Intelectual 2002-2015, INAPI.

A la luz de los resultados (Fig. 7) se puede en una primera instancia determinar que la geografía de la producción de artículos científicos y solicitudes de patentamiento, tomados aquí como indicadores parciales de producción y aplicación de conocimiento, presentan marcadas y sostenidas cumbres y valles. Junto a ello se detecta algún grado de especialización de las FUA, aunque no de forma clara y definitiva; por ejemplo, Puerto Montt es la única que se yergue como Emergente aplicadora de conocimiento. Otras se quedan en la producción de conocimiento, pero son débiles en buscar nuevas aplicaciones para este, como los casos de Arica, La Serena, Talca, Temuco, Valdivia y Punta Arenas. También están las Cumbres, Valparaíso y Concepción, excluyendo Antofagasta, la que, si bien llegó a la categoría, habrá que esperar a ver si es posible que se mantenga o al igual que Puerto Montt se posicione como Emergente aplicadora de conocimiento, en línea con su importante desarrollo industrial extractivo.

Finalmente, Santiago, pese a su estabilidad como Súper cumbre, devela una relativa pérdida de competitividad, especialmente en su condición de FUA aplicadora de conocimiento, 
Figura 6. Gráfico categorización FUA. Fuente: elaboración

propia sobre la base de datos catastrados, años 2002-2015

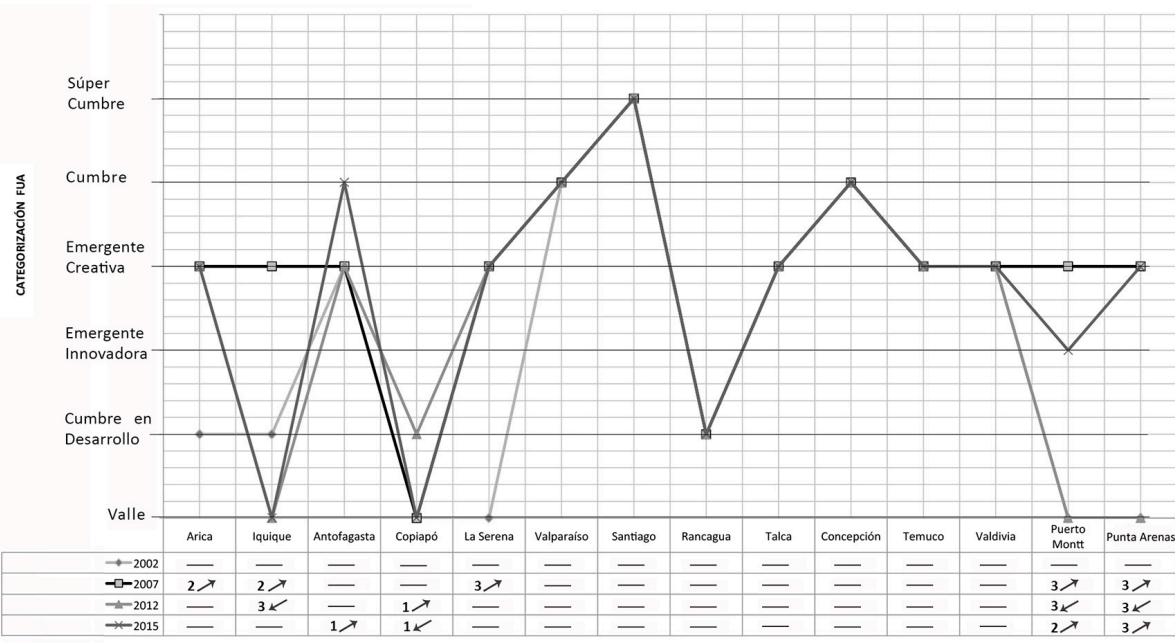

\begin{tabular}{|c|c|c|c|c|c|c|c|c|c|c|c|c|c|c|}
\hline CATEGORIA 2002 & Arica & Iquique & Antofagasta & Copiapó & La Serena & Valparaiso & Santiago & Rancagua & Talca & Concepción & Temuco & Valdivia & $\begin{array}{l}\text { Puerto } \\
\text { Montt }\end{array}$ & Punta Arenas \\
\hline \multicolumn{15}{|l|}{ SÚPER CUMBRES } \\
\hline \multicolumn{15}{|l|}{ CUMBRES } \\
\hline \multicolumn{15}{|c|}{ EMERGENTES CREADORAS } \\
\hline \multicolumn{15}{|c|}{ EMERGENTES APLICADORAS } \\
\hline \multicolumn{15}{|c|}{ CUMBRES EN DESARROLLO } \\
\hline \multicolumn{15}{|l|}{ VALLES } \\
\hline CATEGORIA 2007 & Arica & Iquique & Antofagasta & Copiapó & La Serena & Valparaiso & Santiago & Rancagua & Talca & Concepción & Temuco & Valdivia & $\begin{array}{l}\text { Puerto } \\
\text { Montt }\end{array}$ & Punta Arenas \\
\hline \multicolumn{15}{|l|}{ SÚPER CUMBRES } \\
\hline \multicolumn{15}{|l|}{ CUMBRES } \\
\hline \multicolumn{15}{|c|}{ EMERGENTES CREADORAS } \\
\hline \multicolumn{15}{|c|}{ EMERGENTES APLICADORAS } \\
\hline \multicolumn{15}{|c|}{ CUMBRES EN DESARROLLO } \\
\hline \multicolumn{15}{|l|}{ VALLES } \\
\hline CATEGORIA 2012 & Arica & Iquique & Antofagasta & Copiapó & La Serena & Valparaíso & Santiago & Rancagua & Talca & Concepción & Temuco & Valdivia & $\begin{array}{l}\text { Puerto } \\
\text { Montt }\end{array}$ & Punta Arenas \\
\hline \multicolumn{15}{|l|}{ SÚPER CUMBRES } \\
\hline \multicolumn{15}{|l|}{ CUMBRES } \\
\hline \multicolumn{15}{|c|}{ EMERGENTES CREADORAS } \\
\hline \multirow{2}{*}{\multicolumn{15}{|c|}{$\begin{array}{l}\text { EMERGENTES APLICADORAS } \\
\text { CUMBRES EN DESARROLLO }\end{array}$}} \\
\hline & & & & & & & & & & & & & & \\
\hline \multicolumn{15}{|l|}{ VALLES } \\
\hline CATEGORIA 2015 & Arica & Iquique & Antofagasta & Copiapó & La Serena & Valparaiso & Santiago & Rancagua & Talca & Concepción & Temuco & Valdivia & $\begin{array}{l}\text { Puerto } \\
\text { Montt }\end{array}$ & Punta Arenas \\
\hline \multicolumn{15}{|l|}{ SÚPER CUMBRES } \\
\hline \multicolumn{15}{|l|}{ CUMBRES } \\
\hline \multicolumn{15}{|c|}{ EMERGENTES CREADORAS } \\
\hline \multirow{2}{*}{\multicolumn{15}{|c|}{$\begin{array}{l}\text { EMERGENTES APLICADORAS } \\
\text { CUMBRES EN DESARROLLO }\end{array}$}} \\
\hline & & & & & & & & & & & & & & \\
\hline VALLES & & & & & & & & & & & & & & \\
\hline
\end{tabular}

$\lessdot \nearrow$ №: Movimiento FUA en el escenario de la innovación. 
donde el indicador se mantuvo estable en el período, a pesar del importante aumento de su PIB, atracción de fondos públicos para investigación y capacidad de producir conocimiento, contrastando con el dinamismo de Concepción, que en el mismo ítem presenta un aumento absoluto y porcentual significativo en esta variable.

Entonces, producto del cruce de variables e interpretación de resultados, se explicitan las FUA que se mueven dentro de un rasgo estructural marcado por la producción de conocimiento, pero también por la falta de competencias para hacer de ese conocimiento nuevas aplicaciones, lo que se hace extensivo a los sistemas Cumbres y Súper cumbre. También es posible ver las FUA que estando fuera del límite inferior del rasgo estructural, pueden ser objeto de intervenciones focalizadas, ya sea con programas o estrategias específicas, tendientes a mejorar su condición de generación de conocimiento o aplicación de este. También se visibiliza un rasgo estructural, cuya modificación ya no pasa por políticas focalizadas, sino más bien por cambios en el ámbito de la política pública de largo plazo.

\section{Consideraciones finales}

En los últimos años han aparecido con cierta abundancia una serie de ranking, indicadores y clasificaciones urbanas, como el Índice de Competitividad Global (https://www.weforum. org/), el Índice de Desarrollo Humano (http://www.cl.undp.org/) o el Índice de Calidad de Vida Urbana (http://www.estudiosurbanos.uc.cl/), por nombrar algunos. Sin embargo, son escasos los ranking o indicadores que den cuenta de la participación de los sistemas urbanos en la llamada economía del conocimiento, por lo que resulta novedosa la propuesta aquí desarrollada, por cuanto sugiere una metodología simple de levantar y leer, evaluando rápidamente el posible impacto del PIB local e inversión pública en investigación sobre las dinámicas de producción y aplicación de conocimiento. Así también identifica los sistemas urbanos que estando bajo la media tienen potencial de ascender, objetos de políticas focalizadas, y los que estando en la media o rasgo estructural, pueden ser apalancados como parte de una política general de emprendimiento.

La principal fortaleza de la metodología se verifica en su fácil levantamiento y seguimiento, siendo un potencial complemento a la evaluación de políticas de desarrollo y competitividad, basadas en incentivos a la investigación y patentamiento. En este contexto, el método de medición propuesto logra interrelacionar y complementar distintos datos, identificar dinámicas de cambio y advertir movimientos significativos que justifiquen un análisis a 
Figura 7.

Clasificación FUA; producción y aplicación de conocimiento, año 20022015. Fuente: elaboración propia sobre la base de datos catastrados, años 2002-2015.

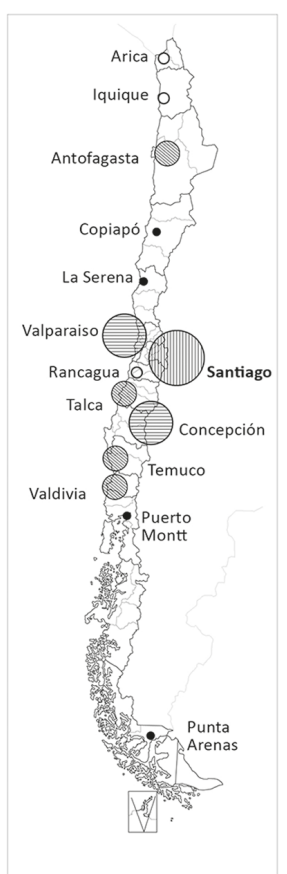

Mapa de Chile, 2002.

\section{SIMBOLOGÍA}



Mapa de Chile, 2007.

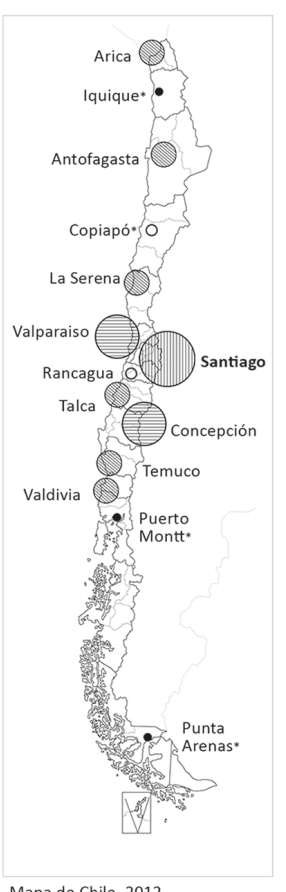

Mapa de Chile, 2012.

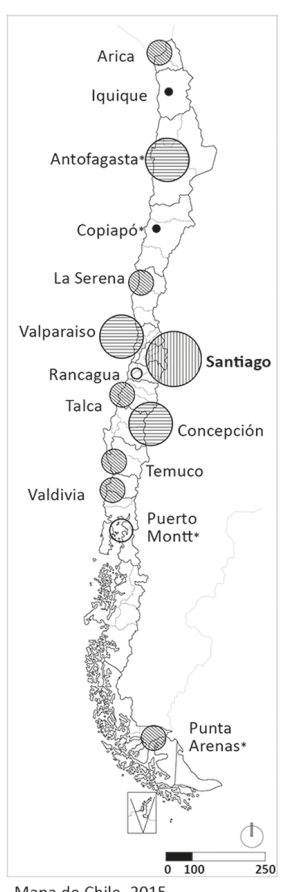

Mapa de Chile, 2015.

mayor profundidad. La debilidad está en la sensibilidad de los indicadores, que no llegan a identificar optimizaciones y pérdidas de eficiencia si no significan un movimiento de la FUA, y sumergen cambios que pueden ser relevantes de exponer, lo que se acentúa con Santiago, que genera una desviación estándar con una banda media de mayor amplitud y en ascenso. Otra debilidad es que no ofrece una explicación a lo que deja en evidencia. 
Pese a lo anterior, la clasificación puede ganar precisión al aumentar las variables, como por ejemplo el Índice de Desarrollo Humano, oferta de empleos o generación de empresas según rubro homologado a clasificación KIS. También puede aumentar sensibilidad, especialmente para las FUA intermedias, si se excluye Santiago, lo que estrecharía el rasgo estructural nacional. Y en lo que respecta a la explicación de lo que arroja, esto necesariamente requiere un estudio profundo caso a caso, que incorpore otras variables de corte preferentemente cualitativo. En segundo término, la aproximación que permite este cruce de datos genera resultados que se expresan de manera comprensible y de fácil interpretación, lo que facilita la divulgación de resultados y la posible interacción con actores relevantes en la toma de decisiones sobre los procesos de articulación entre generadores de conocimiento, emprendedores y política pública. En tercer lugar, queda en evidencia que integrar variables de orden económico y demográfico con otras vinculadas con la producción y aplicación de conocimiento se vuelve apropiado para un país como Chile, que pese a una vigorosa y territorialmente concentrada economía neo-extractivista primaria, evidencia señas de agotamiento y difícil integración de valor agregado, lo que hace necesario pensar en políticas sostenibles de reconversión productiva, con miras a la consolidación de servicios intensivos en conocimiento, que potencien y proyecten el saber hacer que se ha venido acuñando a partir de la industrialización de mediados del siglo XX hasta la actualidad.

Finalmente, la concentración geográfica de población y generación de riqueza son importantes en el momento de explicar la producción y aplicación de conocimiento (FLoRidA, 2009); sin embargo, por lo menos para los casos y períodos revisados, esto no se cumple del todo. Entonces -y especialmente en aquellos casos que están fuera de la media- entra como potencial explicación la propia FUA y sus características territoriales, historia, contexto geográfico, singularidades sociales, estructura y morfología urbana.

¿Qué ocurre en aquellas FUA que, teniendo altos indicadores de población, PIB, atracción de recursos para investigación y producción de conocimiento, no son capaces de generar nuevas aplicaciones del conocimiento que producen? Una eventual e inicial respuesta puede estar en la propia FUA y sus condiciones internas de proximidad e integración física, social y funcional, necesarias para transferir y difundir fácilmente el conocimiento, pero también para generar encuentro entre quienes lo crean con los que buscan y financian nuevas aplicaciones para este, haciendo de las ideas innovaciones (Katz \& BradLey, 2015; JACobs, 2011). 
Una vez analizados los datos que aquí se produjeron e identificadas las singularidades que escapan a la media, tanto en el tramo superior como en el inferior, queda abierta la interrogante sobre el papel que juegan las FUA y sus características organizacionales, estructurales, funcionales y morfológicas en la sostenibilidad y descentralización de la economía del conocimiento, y el surgimiento de ecosistemas locales amplios que nutran y fomenten el conocimiento y la creatividad. Claves, por lo menos en la literatura internacional, resultan ser la densidad poblacional, mixtura de usos, cultura, recreación, sociedades tolerantes y diversas y servicios urbanos variados, ya que dan las condiciones para la permanente rúbrica y ampliación de las redes formales e informales de información, basadas en la interacción persona a persona (Castells \& Hall, 2001; Gertler, Geddie, Hatch \& Rekers, 2014; Katz \& Bradley, 2015).

\section{Bibliografía}

AGUILAR, A. (2002). "Megaurbanization and Industrial Relocation in Mexico Central Region”. Urban Geography, 23 (7).

BRENNER, N. \& THEODORE, N. (2002) (eds.) Space of Neoliberalism: Urban Restructuring in North America and Western Europe. Oxford: Blackwell.

CASTELLS, M. \& HALL, P. (2001) Tecnópolis del mundo. La formación de los complejos industriales del siglo XXI. Alianza Editorial. Madrid, España.

CHION, M. (2002). "Dimensión metropolitana de la globalización: Lima a fines del siglo XX". En: EURE (Santiago), 28 (85), 71-87. Recuperado el 23 de mayo de 2016.

CICCOLELLA, P. (1999). "Globalización y dualización en la Región Metropolitana de Buenos Aires: Grandes inversiones y reestructuración socioterritorial en los años noventa”. En EURE (Santiago), 25 (76).

COSTAS, P. (2008). "Creativity, innovation and territorial agglomeration in cultural actvities: Theroots of the creative city". En Creative cities, cultural clusters and local economic development, ed. Philip Cooke and Luciana Lazzeretti. Cheltenham, UK: Edward Elgar Publishing Ltd. DARCHEN, S. \& TEMBLAY, D. (2014). "Attracting Know ledge Workers and Creative City Paradigm: can We Plan for Talent in Montreal?”. In Seeking Talent for Creative Cities. Ed. Grant, J. University of Toronto Press. Toronto, Canada.

DE MATTOS, C. (2010). "Globalización y metamorfosis metropolitana en América Latina: De la ciudad a lo urbano generalizado”. En Rev. Geografía Norte Grande, 47.

DUCCI, M. (2002). "Santiago: territorios, anhelos y temores. Efectos sociales y espaciales de la expansión urbana". En "Santiago en EURE. Huellas de una metamorfosis metropolitana 1970 / 2000”. En: EURE (Santiago), 32 (97). 
ESCOLANO, S.; ORTIZ, J. \& MORENO, R. (2007). "Globalización y cambios funcionales recientes en las ciudades del sistema urbano chileno”. En Cuadernos Geográficos, vol. 41, N. $^{\circ} 2$.

ESCOLANO, S. \& ORTIZ, J. (2010). "Desarrollo de la viticultura y servicios para la producción en el valle de Copiapó (Chile) en el contexto de la mundialización económica”. En Revista de Geografía Norte Grande, 46.

EUROSTAT REGIONAL YEAR BOOK (2014) Luxembourg: Publications Office of the European Union.

FLORIDA, R. (2009) Por qué donde vives puede ser la decisión más importante de tu vida. Ed. Paidós, Barcelona, España.

FLORIDA, R. (2010) La clase creativa. La transformación de la cultura del trabajo y el ocio en el siglo XXI. Ed. Paidós, Barcelona, España.

FLORIDA, R. (2011) El Gran Reset. Nuevas formas de vivir y trabajar para impulsar la prosperidad. Ed. Paidós, Barcelona, España.

FUENTES, L. (2012). "Marco conceptual: globalización, competitividad urbana y estrategias urbanas”. En Santiago de Chile: una ciudad atractiva para un país competitivo. Colección Estudios Urbanos UC. Santiago, Chile.

GARCÍA, B. (2008). "Política cultural y regeneración urbana en las ciudades de Europa occidental: lecciones aprendidas de la experiencia y perspectivas para el futuro". En Revista de Investigaciones Políticas y Sociológicas, 7 (001).

GERTLER, M. GEDDIE, K. HATCH, C. \& REKERS, J. (2014). "Attracting and RetainingTalent: Evidence from Canada's City-Regions”. In Seeking Talent for Ceative Cities. Ed. Grant, Jill. University of Toronto Press, Toronto, Canada.

GONZÁLEZ, D. (2012). "Competitividad y estrategias de desarrollo". En Santiago de Chile: una ciudad atractiva para un país competitivo. Editores De Mattos, C. Bannen, P. \& Fuentes, L. Colección Estudios Urbanos UC. Santiago, Chile.

HALL, P. (1996) Ciudades del mañana. Historia del urbanismo en el siglo XXI. Ed. Del Serbal, Barcelona, España.

HARVEY, D. (2005) A Brief History of Neoliberalism. Oxford: Oxford University Press.

HARVEY, D. (2007) Espacios del Capital, hacia una geografía critica. Ed. Akal, Madrid, España.

HARVEY, D. (2008) Paris, Capital de la Modernidad. Ed. Akal, Madrid, España.

HOWKINS, J. (2001) The Creative Economy: How People Make Money from Ideas. London, New York: Penguin Press. 
HOYMAN, M. \& FARICY. C. (2009). "It Takes a Village: A Test of the Creative Class, Social Capital and Human Capital Theories”. En Urban Affairs Review, 44.

JACOBS, J. (2011) Muerte y vida de las grandes ciudades. Capitán Swing Libros. Madrid, España.

JANOSCHKA, M. (2002). "Urbanizaciones privadas en Buenos Aires: ¿hacia un nuevo modelo de ciudad latinoamericana?”. Cabrales, L. F. (ed.), Latinoamérica: Países abiertos, ciudades cerradas. Guadalajara: Universidad de Guadalajara/UNESCO.

JURAN, J. M., GODFREY, A. B., HOOGSTOEL, R. E., \& SCHILLING, E. G. (1999) Juran's Quality Handbook (Fifth Edition). New York: The McGraw-Hill Companies, Inc.

JURARTE, C. (2011). "Mapping Vilnius as creative city”. LIMES: Borderland Studies, 4 (1).

KATZ, B. \& BRADLEY, J. (2015) The Metropolitan Revolution. How Cities and Metros Area Fixing Our Broken Politics and Fragile Economy. Ed. The Brookings Institution. Washintong, United State.

KRÄTKE, S. (2011) The Creative Capital of Cities: Interactive Knowledge Creation and the Urbanization Economies of Innovation. Wiley-Blackwell.

LANDRY, C. (2008) The Creative City. A tollkit for Urban Innovators. Taylor \& Francis Group. Gateshead, UK.

LÓPEZ LEVI, L. (1997). "Los centros comerciales como espacios multifuncionales”. Argumentos, 27.

LUDEÑA, Wiley (2002). "Lima: poder, centro y centralidad: Del centro nativo al centro neoliberal”. EURE (Santiago), 28 (83). Recuperado el 23 de mayo de 2016.

DE MATTOS, C. (2010). “Globalización y metamorfosis metropolitana en América Latina: De la ciudad a lo urbano generalizado”. En Revista geografía Norte Grande (47).

MARTINELLI, F. y CAVOLA, L. (2002). "The influence of regional demand and institutions on the role of KIS”. En Wood, P. (Ed.) (2001) Consultancy and Innovation. Routledge, London.

MÉNDEZ, R. et ál. (2006). "Redes socio-institucionales e innovación para el desarrollo de las ciudades intermedias”. En Ciudad y Territorio Estudios Territoriales, 38 (148).

MÉNDEZ, R. et ál. (2012). "Economía creativa y desarrollo urbano en España: una aproximación a sus lógicas espaciales”. En EURE. Revista Latinoamericana de Estudios Urbanos y Regionales, 38 (113).

MEYER, K. y BÄHR, J. (2001). "Condominios in Greater Santiago de Chile and their Impact on the Urban Structure”. Die Erde, 132 (3).

MOLINÍ, F. y ESTRADA, E. (2015). "La innovación y la importancia de la cercanía o la leja- 
nía: el caso de Microsoft Consulting Services”. En Revista electrónica de geografía y ciencias sociales, 14 (524).

MULLER E. \& ZENKER A. (2001). "Business services as actors of know ledge transformation: the role of KIBS in regional and national innovation systems”. En Research Policy, Vol. 30.

NAPADENSKY, A. (2014). "(Re)organización funcional y morfologías metropolitanas emergentes en ciudades intermedias latinoamericanas: El caso del Área Metropolitana de Concepción, Chile”. En Anales Seminario Internacional Red Iberoamericana de Investigadores sobre Globalización y Territorio, RII. http://www.rii.sei.ba.gov.br/anais_xiii/gt6/ GT6_AARON.pdf.

NAPADENSKY, A. (2016). "Centros tradicionales, nuevas centralidades y descentralización en metrópolis intermedias latinoamericanas: caso del Gran Concepción, Chile”. En Cuaderno urbano 21 (21).

OCDE (2013) Definition of Functional Urban Areas (FUA) for the OECD metropolitan data base.

PARNREITER, C. (2002). "Ciudad de México: el camino hacia una ciudad global”. En EURE (Santiago), 28 (85).

PARNREITER, C. (2005). "Tendencias de desarrollo en las metrópolis latinoamericanas en la era de la globalización: los casos de Ciudad de México y Santiago de Chile”. En EURE (Santiago), v. 31, n. 92.

PARNREITER, C. (2011). "Formación de la ciudad global, economía inmobiliaria y transnacionalización de espacios urbanos. El caso de Ciudad de México". En EURE (Santiago), 37 (111), 5-24.

PECK, J. (2005). "Struggling with the creative class". En International Journal of Urban and Regional Rsearch, 24 (4).

PÉREZ, C. (2004) Revoluciones tecnológicas y capital financiero. Siglo XXI, Buenos Aires, Argentina.

PÉREZ, C. y MARMOLEJO, C. (2008). "La localización intrametropolitana de las actividades de la innovación: un análisis para la región metropolitana de Barcelona”. En Revista electrónica de geografía y ciencias sociales, 12 (270).

PRATT, A. (2008). "Creative cities: the cultural industries and the creative class”. En Geografiska annaler: Series B - Human geography, 90 (2).

RODRÍGUEZ-POSE, A. y COMPTOUR, F. (2012). "Clusters generate greater innovation and growth? Ananalysis of European regions”. En The professional Geographer, 64 (2).

SÁNCHEZ, S. (2012). "El empleo en la economía del conocimiento en España: características socio-profesionales y patrones de distribución territorial”. En Revista electrónica de geografía y ciencias sociales, 16 (399). 
ScoTT, A. (2006). “CreativeCities: Conceptual Issues and Policy Questions”. En Journal of Urban Affairs, 28, (1).

THROSBY, D. (2004). "Assessing the Impacts of a Cultural Industry. Journal of Arts Management”. Law and Society, 34 (3).

THROSBY, D. (2008). "Modelling the cultural industries". En International Journal of Cultural Policy. 14 (3).

VENCE, X. y RODIL, O. (2003). "La concentración regional de la política de I+D de la Unión Europea: el principio de cohesión en entredicho”. En Revista de Estudios Regionales. 65.

VENCE, X. (2007). "La tercerización y metropolización de la economía mundial: una aproximación a partir de las tendencias en la Unión Europea”. En IX Reunión de Economía Mundial. Madrid. 